\title{
Synthetic aperture radar (SAR)-based mapping of volcanic flows: Manam Island, Papua New Guinea
}

\author{
J. K. Weissel ${ }^{1}$, K. R. Czuchlewski ${ }^{1,2}$, and Y. Kim ${ }^{3}$ \\ ${ }^{1}$ Lamont-Doherty Earth Observatory of Columbia University, Palisades, NY, USA \\ ${ }^{2}$ Department of Earth and Environmental Sciences, Columbia University, New York, USA \\ ${ }^{3}$ Jet Propulsion Laboratory, California Institute of Technology, Pasadena, CA, USA
}

Received: 3 July 2003 - Revised: 3 February 2004 - Accepted: 5 March 2004 - Published: 19 April 2004

\begin{abstract}
We present new radar-based techniques for efficient identification of surface changes generated by lava and pyroclastic flows, and apply these to the 1996 eruption of Manam Volcano, Papua New Guinea. Polarimetric L- and P-band airborne synthetic aperture radar (SAR) data, along with a C-band DEM, were acquired over the volcano on 17 November 1996 during a major eruption sequence. The Lband data are analyzed for dominant scattering mechanisms on a per pixel basis using radar target decomposition techniques. A classification method is presented, and when applied to the L-band polarimetry, it readily distinguishes bare surfaces from forest cover over Manam volcano. In particular, the classification scheme identifies a post-1992 lava flow in NE Valley of Manam Island as a mainly bare surface and the underlying 1992 flow units as mainly vegetated surfaces. The Smithsonian's Global Volcanism Network reports allow us to speculate whether the bare surface is a flow dating from October or November in the early part of the late-1996 eruption sequence. This work shows that fully polarimetric SAR is sensitive to scattering mechanism changes caused by volcanic resurfacing processes such as lava and pyroclastic flows. By extension, this technique should also prove useful in mapping debris flows, ash deposits and volcanic landslides associated with major eruptions.
\end{abstract}

\section{Introduction and background}

The island of Manam $\left(4.1^{\circ} \mathrm{S}, 145.1^{\circ} \mathrm{E}\right)$ is one of Papua New Guinea's most active volcanos (Palfreyman and Cooke, 1976). The $10 \mathrm{~km}$-wide island is located about $16 \mathrm{~km}$ off the northeast coast of Papua New Guinea (P.N.G., Fig. 1). Four large radial valleys, spaced approximately $90^{\circ}$ apart, extend from the unvegetated summit of the conical 1800 m-high stratovolcano to its lower flanks (inset, Fig. 1). These "avalanche valleys" channel lava flows and pyroclas-

Correspondence to: J. K. Weissel

(jeffw@1deo.columbia.edu) tic avalanches that reach the coast during major eruptions. There are two summit craters, named Main and South (inset, Fig. 1), and both are active. In historical times, eruptions have been recorded at frequent intervals since 1616 (e.g. Palfreyman and Cooke, 1976). During the past century, most of the eruptions have originated from South crater, concentrating eruptive products into the SE avalanche valley.

During November 1996, as the NASA PacRim I airborne SAR (AIRSAR) mission was being flown in the southwest Pacific, Manam volcano entered its largest eruption sequence since 1992 (Fig. 2). On 17 November, the DC-8 aircraft, which was operating over northern Australia and Papua New Guinea, was diverted to collect two flightlines of data over the erupting volcano. The eruption culminated in a climactic phase on the 3 December, two weeks after the AIRSAR data acquisition (Fig. 2). Many large pyroclastic flows swept down the SE and SW valleys from south crater, causing 13 deaths (Smithsonian Institution, 1996) and forcing the evacuation of thousands.

The AIRSAR data acquired over Manam Island include full polarimetry at L- and P-band $(0.25 \mathrm{~m}$ and $0.64 \mathrm{~m}$ wavelengths, respectively) and vertically polarized backscatter at C-band (0.06 m wavelength), as well as a digital elevation model (DEM) obtained from single-pass, across-track interferometry at C-Band. For a given radar wavelength, SAR polarimeters measure amplitudes and phases for all possible combinations of polarized backscatter; $h h, h v, v h$ and $v v$, that can be received by a radar antenna capable of sending and receiving horizontally $h$ and vertically $v$ polarized waves. A complete description of the AIRSAR instrument and SAR data acquisition modes can be found at the web site http://airsar.jpl.nasa.gov. In this paper, we analyze polarimetric SAR backscatter from one of the Manam Island flightlines, which was flown along a $135^{\circ}$ heading as the radar "looked" to the northeast across the Island (Fig. 1). The objective of our analysis is to show that scattering mechanism signatures obtained from inversion of the L-band polarimetry provide a useful way to distinguish recent or fresh lava flows from those of previous eruptions. The longer-term goal of 


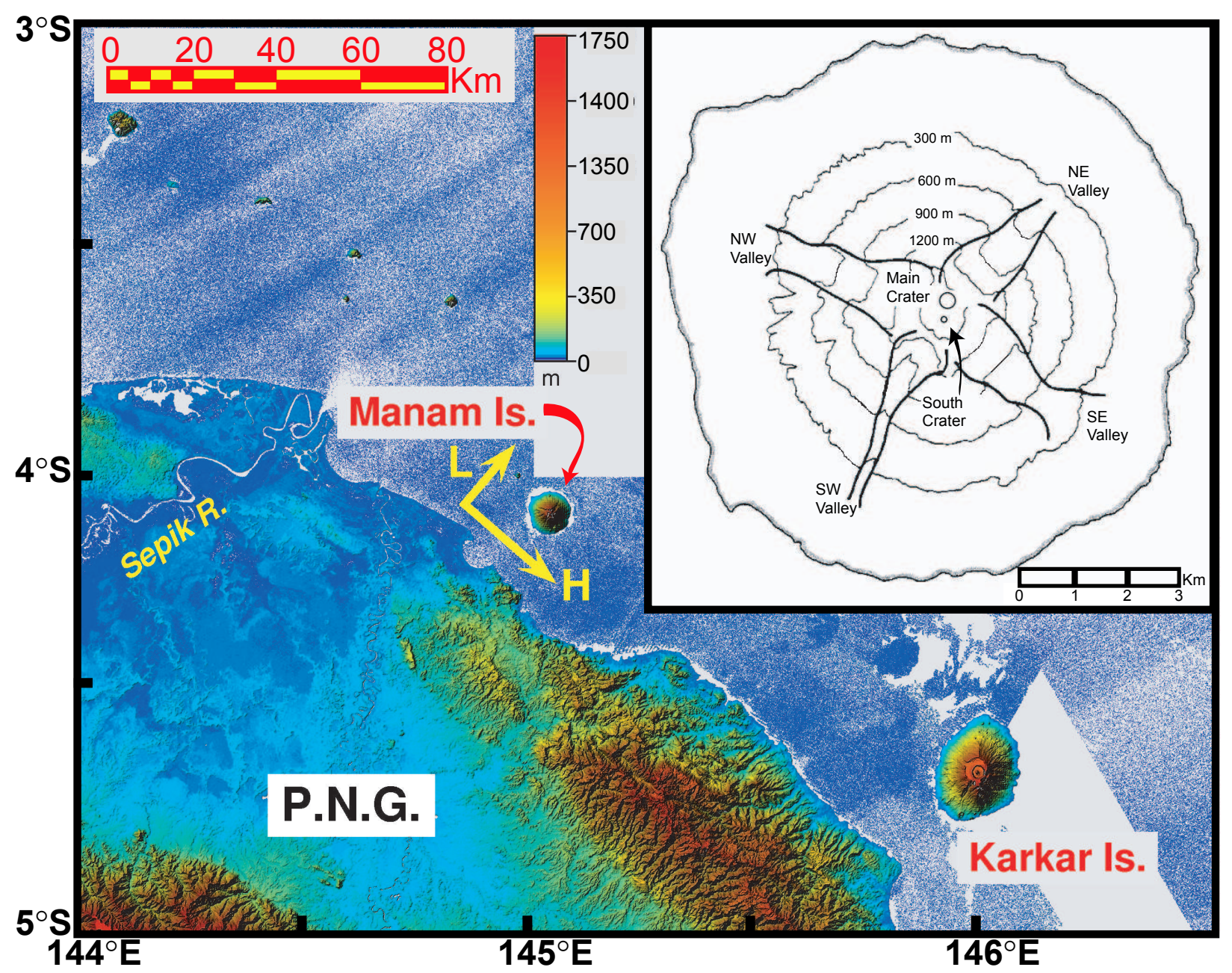

Fig. 1. Location map of Manam Island, off the northeast coast of Papua New Guinea (P.N.G.). Color topography image is from the $90 \mathrm{~m}$ resolution Shuttle Radar Topography Mission (SRTM) data. Flight direction and look direction of the NASA/JPL airborne SAR (the AIRSAR) are denoted by the yellow arrows labeled " $H$ " and "L", respectively. Inset is a sketch topographic map of the island (after Palfreyman and Cooke, 1976) showing the four major valley conduits for lava and pyroclastic flows. Eruptions occur from one of two summit craters, Main and South.

this work is to determine whether polarimetric SAR data and technology might play a significant role in rapid identification and mapping of lava and pyroclastic flows during and after disastrous volcanic eruptions.

During volcanic eruptions in humid climate settings, such as at Manam, vegetated surfaces are replaced by rough, bare surfaces associated with lava and pyroclastic flows (e.g. Fig. 3). Radar scattering mechanisms will therefore change from primarily volume scattering from vegetation to primarily single-bounce scattering from bare surfaces. With time following eruption the flow surfaces will become revegetated. Because our primary interest is rapid mapping of lava and pyroclastic flows from disastrous eruptions using SAR data and technology, we focus on methods that efficiently discriminate between backscatter properties of bare and vegetated surfaces. Our expectation is that relatively fresh or recent flows (e.g. Fig. 3) will be characterized by scattering signatures characteristic of rough, bare surfaces.

\section{Inversion of SAR polarimetry for scattering mechanism signatures}

Radar microwave signals interact with scattering objects (such as trees, bare surfaces, etc.) in different ways depending on the shape and electrical characteristics of these objects. It is therefore possible that the shape and electrical characteristics of surface materials can be inferred from SAR backscatter. For an electromagnetic wave that is both transverse and polarized, a $2 \times 2$ matrix $S$ of complex scattering coefficients $S_{p q}$ determines the scattering properties (in an "average" sense) of materials within a SAR resolution cell $(10 \mathrm{~m} \times 10 \mathrm{~m}$ for the Manam data). The scattering matrix $S$ linearly relates the incident electric field $\boldsymbol{E}^{\boldsymbol{i}}$ to the backscattered electric field $\boldsymbol{E}^{s}$ received at the radar antenna:

$\left[\begin{array}{c}E_{h}^{s} \\ E_{v}^{s}\end{array}\right]=\frac{e^{i k r}}{r}\left[\begin{array}{ll}S_{h h} & S_{h v} \\ S_{v h} & S_{v v}\end{array}\right]\left[\begin{array}{c}E_{h}^{i} \\ E_{v}^{i}\end{array}\right]$ 

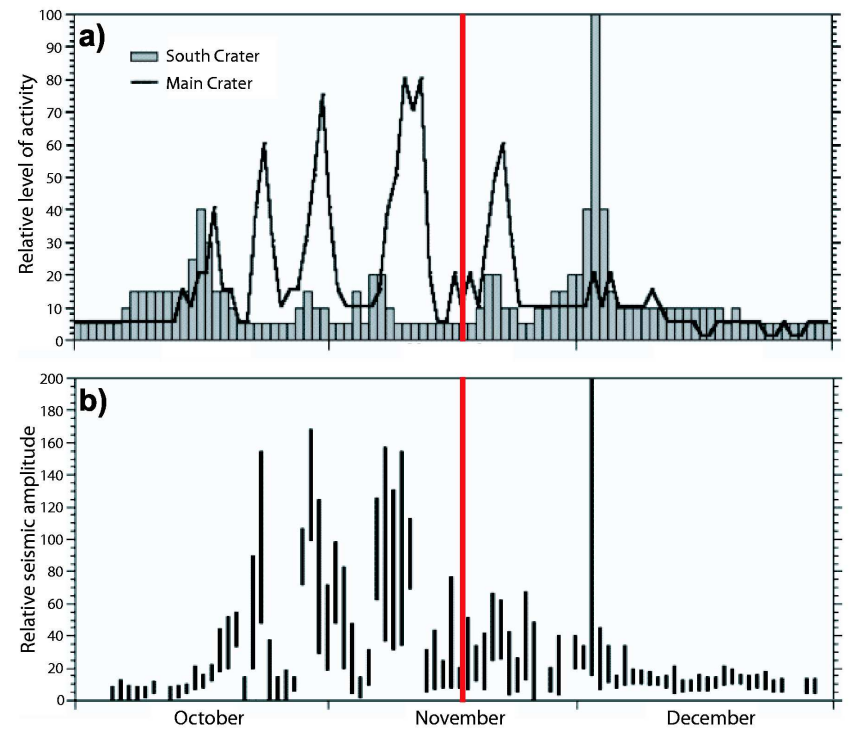

Fig. 2. (a) Indices of volcanic activity at the Main and South craters, Manam Island, for the eruptive sequence October - December 1996. (b) Indices showing relative level of seismic activity during the late1996 eruptive sequence. Red vertical line marks date of AIRSAR acquisition over Manam Island. Data courtesy of the Rabaul Volcano Observatory. Source: Smithsonian Institution (1996).

(e.g. Nghiem et al., 1990; Boerner et al., 1998). In Eq. (1), which is written explicitly in terms of horizontal $h$ and vertical $v$ components of the electric fields, $k$ denotes the wavenumber of the illuminating wave and $r$ is the distance of the target from the radar. The elements $S_{p q}$ of $S$ depend on the geometry, roughness and electrical properties of the target material, as well as on the polarization state (in the $h-v$ reference frame) of the incident wave. For monostatic SAR measurements (one antenna for both transmission and reception), we may take $S_{h v}=S_{v h}$ in Eq. (1) because of reciprocity (Huynen, 1965); thus only three elements of $\boldsymbol{S}$ are required to describe scattering.

As for all inverse problems we need to ask whether a scattering matrix uniquely defines a scattering object. Unfortunately, in general the answer is "no." Even though a unique solution is not guaranteed, this inverse problem may be meaningfully "solved" for some simple applications for which sufficient constraints are available. We have described elsewhere (Czuchlewski et al., 2003) general methods for inversion of SAR polarimetry to identify and map landslides in the humid, tectonically active mountains of Taiwan. Such methods are based on the radar target decomposition techniques described by Cloude and Pottier $(1996,1997)$. Because our purpose is rapid detection of surface changes caused by lava and pyroclastic flows on Manam Island, we develop a simple classification scheme that efficiently discriminates the rough bare surfaces of recent flows from surrounding vegetation (e.g. Fig. 3).

We first express target scattering properties on a per-pixel basis in terms of a complex vector $\boldsymbol{t}$, which is formed by pro-

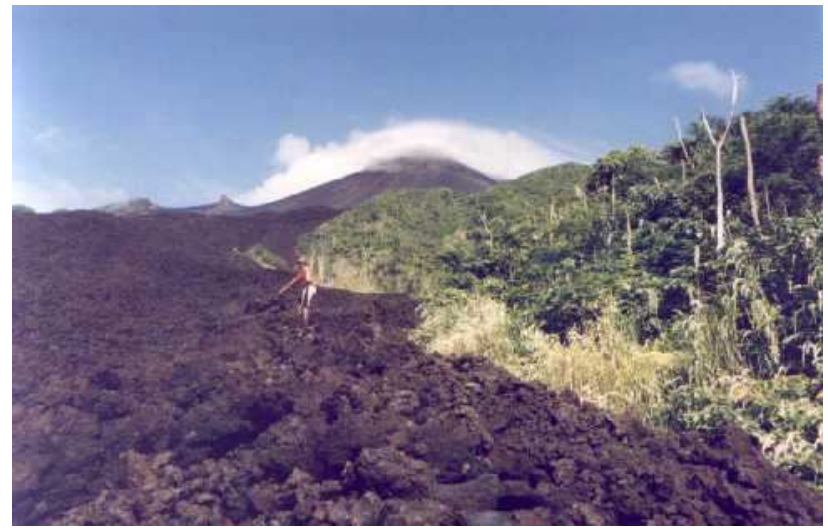

Fig. 3. Hand-held photograph taken by Nir Halman of Tel Aviv University, Israel, in November 1995 shows a lava flow erupted in SE Valley during October 1994 (Smithsonian Institution, 1994) juxtaposed against native scrub (reproduced with permission of the photographer). The rough, 'a'a-like flow surface has remained unvegetated for a year since eruption.

jecting the scattering matrix $S$ in Eq. (1) on to $2 \times 2$ orthogonal basis matrices chosen to preserve a simple linear ordering of the scattering coefficients (Cloude and Pottier, 1996):

$\boldsymbol{t}=\left[\begin{array}{lll}S_{h h} & \sqrt{2} S_{h v} & S_{v v}\end{array}\right]^{T}$,

where ${ }^{T}$ signifies the transposed matrix, and the factor $\sqrt{2}$ is used to conserve total backscattered power.

Correlations among the elements of the scattering vector are explored by forming the covariance matrix $\mathrm{C}$ of $\boldsymbol{t}$ :

$\mathrm{C}=\left\langle\boldsymbol{t} \boldsymbol{t}^{+}\right\rangle=\left[\begin{array}{ccc}\left\langle S_{h h} S_{h h}^{*}\right\rangle & 0 & \left\langle S_{h h} S_{v v}^{*}\right\rangle \\ 0 & 2\left\langle S_{h v} S_{h v}^{*}\right\rangle & 0 \\ \left\langle S_{h h} S_{v v}^{*}\right\rangle^{*} & 0 & \left\langle S_{v v} S_{v v}^{*}\right\rangle\end{array}\right]$,

where $^{*}$ is the conjugate, ${ }^{+}$the conjugate of the transposed matrix, and \langle\rangle indicates ensemble averaging. The zero elements in Eq. (3) indicate that we explicitly assume reflection symmetric media, and the co- and cross-polarized scattering coefficients will therefore be uncorrelated, i.e. $\left\langle S_{h h} S_{h v}^{*}\right\rangle=\left\langle S_{v v} S_{h v}^{*}\right\rangle=0$ (Nghiem et al., 1992). The remaining elements of the covariance matrix are related to normalized radar cross-sections and correlation coefficients $\sigma_{p q r s}$ :

$\sigma_{p q r s}=\lim _{A_{c} \rightarrow \infty} \frac{4 \pi}{A_{c}}\left\langle S_{p q} S_{r s}^{*}\right\rangle$,

where indices $p, q, r, s$ stand for either horizontal $h$ or vertical $v$ polarizations, and $A_{c}$ is radar illumination area (Nghiem et al., 1992). In particular, when $p=r$ and $q=s$, we obtain the co- and cross-polarization radar cross-sections from the elements on the leading diagonal of the covariance matrix $\boldsymbol{C}$ in Eq. (3).

Next, an eigenvalue/eigenvector decomposition is performed on the covariance matrix $C$ :

$\boldsymbol{C}=\lambda_{1} \kappa_{1} \cdot \kappa_{1}^{+}+\lambda_{2} \kappa_{2} \cdot \kappa_{2}^{+}+\lambda_{3} \kappa_{3} \cdot \kappa_{3}^{+}$. 
This allows us to partition a SAR resolution cell's scattering properties into as many as three orthogonal (or independent) scattering mechanisms given by the eigenvectors $\kappa_{i}$, whose relative importance is determined by the eigenvalues $\lambda_{1} \geq \lambda_{2} \geq \lambda_{3}$. The challenge lies in relating the scattering mechanisms represented by the eigenvectors to polarimetric backscatter from common surface cover types like rough surfaces, water bodies, vegetation, etc.

Our classification method, which was introduced by Kim and van Zyl (2001), is based on decomposition of the covariance matrix as described by Eqs. (1-5). We aim to classify three types of terrain: bare land surfaces, the ocean surface, and forest. Surfaces that do not clearly fall into one of these three terrain types are classified as "ambiguous".

\subsection{Ocean surface}

Radar signatures of water surfaces are dominated by Bragg scattering, which is a resonance effect between the Fourier components of the surface relief and the wavelength and incidence angle of the incident wave (e.g. Valenzuela, 1978). Ocean surface roughness at radar wavelength scales is primarily due to gravity - capillary waves that are generated by wind shear. Sea surface roughness will increase with wind speed. These "Bragg" waves may be modulated at longer wavelengths by swell and waves. Surfaces of shallow, fresh water bodies roughened by swift currents also exhibit Bragg-like scattering properties, as we have shown in Taiwan (Czuchlewski et al., 2003).

Slightly rough water surfaces are identified using conditions for the polarization cross section and cross correlation elements of the covariance matrix $\boldsymbol{C}$ in Eq. (4):

$\Re\left(\sigma_{h h v v}\right)>\sigma_{h v h v}$, and $\sigma_{v v v v}>\sigma_{h h h h}$,

where $\Re$ means the real part of a complex variable. Three scattering properties are implied in the above conditions. First, the co-polarization components are in phase, indicating single-bounce scattering. Second, the co-polarization backscatter cross sections are much larger than the crosspolarization counterpart, indicating little depolarization. Third, the vertical backscatter cross section is always larger than the horizontal cross section, in accordance with Bragg scattering theory (Valenzuela, 1978).

\subsection{Bare land surfaces}

Scattering from bare land surfaces is not necessarily governed by the same physics that determines backscatter from water surfaces. At Manam Island, for example, the bare lava and pyroclastic flow surfaces that replace vegetation are often very rough at radar wavelength scales. This is the case for the 'a'a-like flow erupted into the lower slopes of the SE Valley in 1994 (Fig. 3), and for the pyroclastic debris aprons found around the two craters near the summit of the volcano. Blocky, rough surfaces produce specular reflections from favorably-oriented facets of coarse debris (Zebker et al., 1987). Accordingly, we drop the second, or Bragg, condition in Eq. (6) from the criteria for backscatter from rough land surfaces, such as lava and pyroclastic flows. By omitting the Bragg condition, we implicitly assume that the horizontal backscatter cross section can equal, or even exceed, the vertical for backscatter from rough, bare surfaces. We found that this was indeed the case for the Tsaoling landslide source area and debris apron studied with L-band polarimetry in Taiwan (Czuchlewski et al., 2003). Backscatter from rough, bare land surfaces may undergo more depolarization compared to backscatter from the sea surface. We use this to discriminate between bare land and sea surfaces by setting pedestal heights $\Phi$ acceptable for the ocean surface to lower values than bare land surfaces (see below).

\subsection{Forest cover}

Areas covered by forest can be found where three polarimetric parameters determined from the radar cross sections in Eq. (4) and the eigenvalues of the decomposition of the covariance matrix $\boldsymbol{C}$ in Eq. (5) have values that exceed empirically-determined thresholds. These parameters are listed below.

1. Radar vegetation index $V$, which is derived from the cross sections, is given by

$V=\frac{8 \sigma_{h v h v}}{\sigma_{t}}$,

where $\sigma_{t}=\sigma_{h h h h}+\sigma_{v v v v}+2 \sigma_{h v h v}$ is the total backscatter power (Kim and van Zyl, 2001). Radar vegetation index weighs the contribution of the crosspolarized returns to total power. It will be relatively high where there is diffuse, volume scattering from structural elements of trees (leaves, twigs, branches, etc.).

2. Scattering entropy $H$, which is found from the eigenvalues in Eq. (5), is given by

$H=\sum_{n=1}^{3}-P_{n} \log _{3}\left(P_{n}\right), \quad 0 \leq H \leq 1$,

where $P_{n}=\lambda_{n} / \sum_{m=1}^{3} \lambda_{m}$, is a measure of the randomness or disorder of scattering (Cloude and Pottier, 1996, 1997). For entropy $H=0$, the covariance matrix $\boldsymbol{C}$ has only one non-zero eigenvalue, and scattering properties within a SAR resolution cell can therefore be described by a single, discrete scattering mechanism. Conversely, for $H=1$, the three eigenvalues in Eq. (5) are equal, indicating no dominant scattering mechanism for that cell, and that the scattering process can be considered random. Entropy will be high for forest cover because the depolarizing effect of volume scatter from vegetation elements tends to randomize the backscattered signal. 
3. Pedestal height $\Phi$ is derived from the eigenvalues of the decomposition of the covariance matrix:

$\Phi=\min \left(\lambda_{1}, \lambda_{2}, \lambda_{3}\right) /\left(\lambda_{1}+\lambda_{2}+\lambda_{3}\right)$.

It varies on a range $0 \leq \Phi \leq 1 / 3$, c.f. Eq. (8). Pedestal height increases with the amount of depolarized energy in the return signal (Zebker et al., 1987). As discussed for the entropy parameter, forest, like most types of vegetation cover, is a depolarizing medium.

Because the three vegetation-sensitive polarimetric parameters all depend on depolarization effects of volume scattering, they appear to be redundant. However, since all SAR data suffer speckle noise, the use of multiple parameters can help reduce deleterious speckle noise effects. That is, if the polarimetric parameters are not correlated, the speckle noise in each is statistically independent. Under these conditions, the use of multiple polarimetric parameters will improve classification results. The amount of improvement depends on the correlation among the polarimetric parameters. For example, for forested areas, the horizontal, vertical, and cross polarization backscatter components are strongly decorrelated. In this case, the benefit of using multiple parameters for vegetation estimation is significant.

For classification purposes, forest covered areas are those satisfying the following criteria using Eqs. (7-9): $V>V_{\text {min }}, \Phi>\Phi_{\text {min }}$, and $H>H_{\text {min }}$; where $V_{\text {min }}, \Phi_{\min }$, and $H_{\min }$ are threshold values determined empirically from areas of known forest cover (Kim and van Zyl, 2001). The threshold values for the vegetation-sensitive polarimetric parameters were established using training areas where the cover type could be identified unambiguously in independent data, such as photography (e.g. Fig. 3).

\subsection{Empirical thresholds for surface cover classification}

We classified the L-band polarimetry obtained on 17 November 1996 over Manam Island (Fig. 4) in terms of bare land surfaces, ocean surface, forest, and "ambiguous" according to the above criteria and threshold values for the polarimetric parameters: vegetation index $V$, entropy $H$, and pedestal height $\Phi$, as follows:

Forest (green): $V>0.6$, and either $H>0.6$ or $\Phi>0.15$.

Ocean surface (blue): Eq. (6), and $\Phi<0.0015$.

Bare land surface (purple): $\Re\left(\sigma_{h h v v}\right)>\sigma_{h v h v}$ in Eq. (6), and $0.0015 \leq \Phi<0.02$.

No data, or ambiguous classification, (black).

\section{Results and discussion}

Figure 4 shows a three dimensional perspective of Manam Island where surface cover type has been classified from the

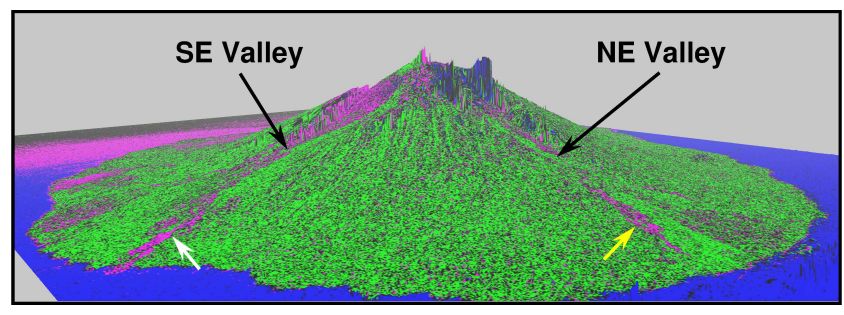

Fig. 4. Three-dimensional perspective image showing L-band surface classification results for Manam Island viewed from the east: Blue - ocean surface; Purple - bare land surfaces; Green forested surface; Black - not classified; using the method and criteria described in the text. White and yellow arrows point to bare land surfaces on the lower slopes of the SE and NE radial valleys that could indicate recent lava flows.

decomposition of the covariance matrix C using L-band polarimetry, together with the threshold criteria for scattering entropy $H$, radar vegetation index $V$, and pedestal height $\Phi$, described above. The selection of the polarimetric parameters and their threshold values is very important for the success of the proposed classification algorithm. In order for the algorithm to be applicable for various SAR sensors and many different terrain types, we have concentrated on "relative" rather than "absolute" polarimetric parameters. Relative polarimetric parameters, such as entropy $H$, radar vegetation index $V$, and pedestal height $\Phi$, use the ratio of absolute polarimetric parameters, such as radar cross sections $\sigma_{p q r s}$ and eigenvalues $\lambda_{m}$. This is done because relative polarimetric parameters are less sensitive to both incidence angle variations and instrument calibration errors since any multiplicative errors will be cancelled (Kim and van Zyl, 2001). Threshold values for each of the vegetation-sensitive relative polarimetric parameters were derived from the AIRSAR data using ground truth information available from Manam Island (e.g. Fig. 3). Previous experience using AIRSAR polarimetry over the Landes forest of southwest France allowed us to establish reasonable ranges for threshold values for use in vegetation mapping. The earlier studies were designed to estimate above-ground biomass for these managed pine forests (e.g. Le Toan et al., 1992). It was found that the relative polarimetric parameters increase as biomass increases from fresh clear-cuts through increasing stand age, so that radar parameter averages for an average stand age could be obtained.

To test the sensitivity of the classification results to variations in threshold values $V_{\min }, H_{\min }$ and $\Phi_{\min }$ given above for the vegetation-sensitive relative polarimetric parameters, we varied the thresholds by $\pm 20 \%$ and repeated the classification procedure. The results are shown in Fig. 5. A $-20 \%$ change to threshold values for radar vegetation index $V$, entropy $H$, and pedestal height $\Phi$ corresponds to the following criteria for Forest cover (green): $H>0.48, V>0.48$ and $\Phi>0.12$; for Ocean (blue): $\Phi<0.0012$; and for Bare Land (purple): $0.0012<\Phi<0.016$. A $+20 \%$ change corresponds to the following thresholds for Forest: $H>0.72, V>0.72$ 


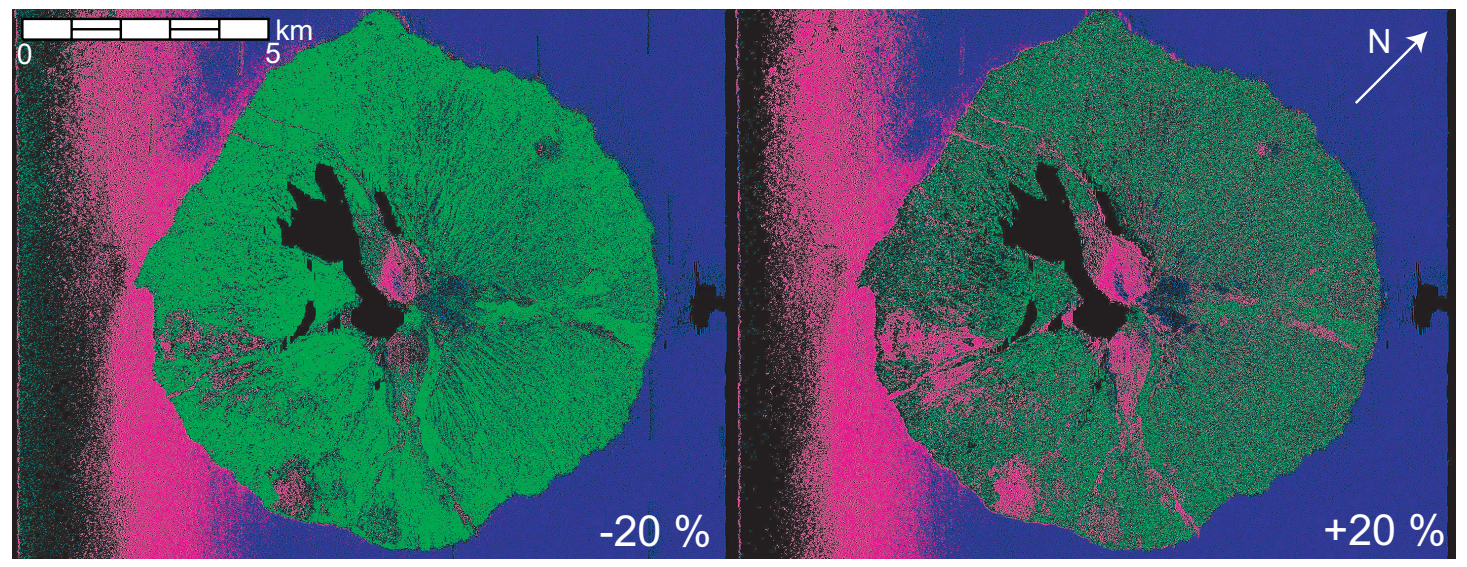

Fig. 5. Classification results when the threshold values $V_{\min }, H_{\min }$, and $\Phi_{\min }$ for the vegetation-sensitive polarimetric parameters vegetation index Eq. (7), entropy Eq. (8), and pedestal height $\Phi$ Eq. (9) are varied by $\pm 20 \%$ from values selected from available ground truth information (see discussion in the text). Note that the near range of the AIRSAR swath is on the left side of the two panels.

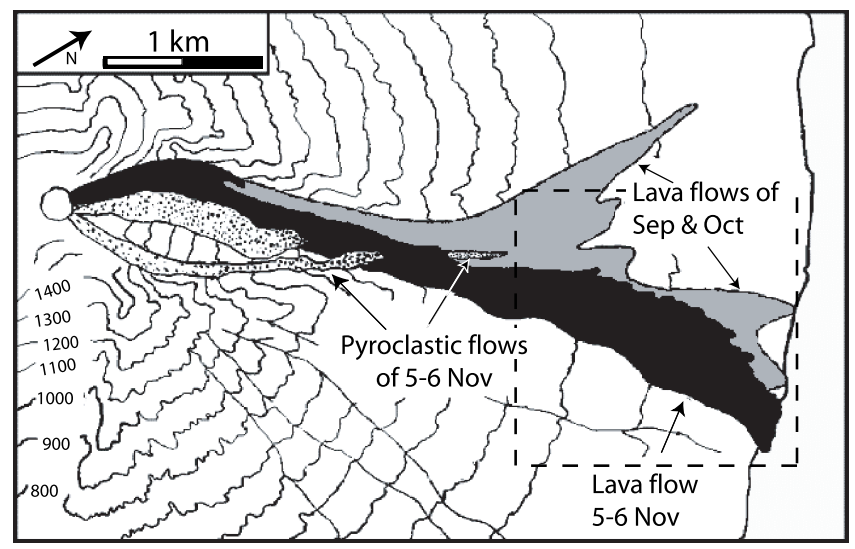

Fig. 6. Map of lava and pyroclastic flows down the NE Valley during the September to November 1992 eruption sequence. Area in dashed box is studied in detail using 17 November 1996 SAR polarimetry and surface classification in Fig. 7. Source: Rabaul Volcanological Observatory for original data, and Smithsonian Institution (1992). Elevation contours are in meters.

and $\Phi>0.18$; for Ocean: $\Phi<0.0018$; and for Bare Land: $0.0018<\Phi<0.024$. Raising the thresholds $(+20 \%)$ means less of the terrain is classified as forest (Fig. 5), and vice versa. We point out that although $40 \%$ represents a large part of the total range of the vegetation-sensitive polarimetric parameters, the classification results do not change appreciably as thresholds are varied over that range (Fig. 5). Most of Manam Island is correctly classified as forest covered (green in Figs. 4 and 5). Likewise, the areal extent of previously classified bare land surfaces vary little as the thresholds are changed, but in the $-20 \%$ case they are now classified as ambiguous. Based on results shown in Fig. 5, we conclude that the proposed algorithm is robust to $\pm 10 \%$ change in the threshold values, and it will work for SAR polarimetry obtained over various terrain types.
The ocean surface (blue) is correctly classified, except at incidence angles less than about $30^{\circ}$ in the near range of the AIRSAR swath (SW region, Figs. 4 and 5), where it is confused with bare land surfaces and, in the most southwestern region of the $-20 \%$ case, forested surfaces. This incidence angle dependence suggests that multiple bounce scattering from the ocean surface is relatively more important in the near range compared to the far range. Multiple bounce also means relatively more depolarization in the near range and therefore a larger value for pedestal height $\Phi$. Because we discriminate between bare land surface and the ocean surface largely on the basis of a pedestal height threshold, if we set the threshold too low $(-20 \%)$, more ocean will be misclassified as bare land (left panel in Fig. 5).

The summit area of the volcano shows bare land surface scattering signatures (purple) consistent with the rocky outcrop in the upper valleys and the extensive pyroclastic debris aprons around the two craters (Figs. 4 and 5). Small areas within the northwestern summit debris apron are misclassified as ocean surface (blue). This possibly reflects deposits of fine ash rather than blocky debris. However, we have no ground truth to support this explanation. Other extremely rough areas in the upper part of NE valley are misclassified as ocean surface. Here, the data quality is very poor, according to the correlation coefficients between the two C-band interferometric channels of the AIRSAR, and any explanation would be suspect.

Of interest to us are the narrow ribbons of bare surface scattering on the lower valley slopes denoted by the white and yellow arrows in Fig. 4. Lava flows on the lower slopes of NE Valley (yellow arrow, Fig. 4) were selected for a more detailed study using the SAR polarimetry and radar scattering mechanisms derived from the L-band data. Field mapping following the large eruption sequence from Main crater in late 1992 delineated two main lava flow units (erupted September - October, and on 5-6 November, respectively; Smithsonian Institution, 1992) in this area, together with smaller pyroclastic flows (Fig. 6). 


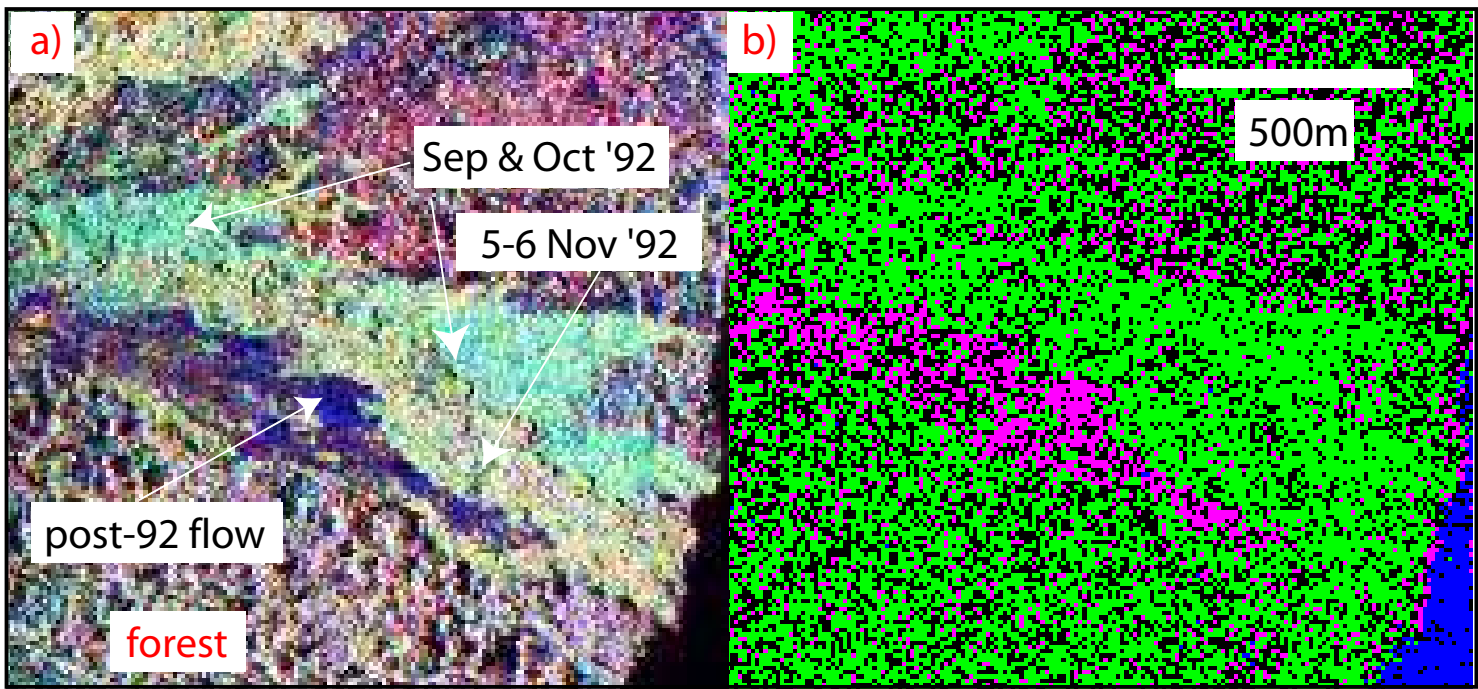

Fig. 7. (a) Phv, Lhv, Cvv RGB composite of the 17 November 1996 AIRSAR data for an area on the lower slopes of the NE Valley (Fig. 6). (b) L-band surface classification results for area shown in (a): Purple - bare land surface, Blue - ocean surface, Green - vegetated surface, Black - not classified, using methods obtain scattering signatures from SAR polarimetry described in the text.

The two 1992 lava flows can be distinguished in the 17 November 1996 AIRSAR data using a Red-Green-Blue (RGB) composite of Phv, Lhv and Cvv (Fig. 7a). The crosspolarization channels of $\mathrm{P}$ - and L-band polarimetry were employed in order to highlight backscatter from vegetation and other depolarizing media. The younger (5-6 November) 1992 flow can be distinguished from the SeptemberOctober flow by its more yellowish hue in the RGB image (Fig. 7a). The boundary between the younger and older flows is delineated by the darker pixels that represent a slight topographic shadow zone on the far-range (northern) edge of the upper flow unit. Note the dark area (low backscatter in Phv, Lhv and Cvv) within the boundaries of the younger, 5-6 November 1992 flow unit (Fig. 7a). This low backscatter unit is classified as bare land surface (Fig. 7b) using the methods described above. One interpretation is that this bare surface is a fresh or recent lava flow. The lobate outline of this scattering unit is consistent with the idea that it is a lava flow that overlies the 5-6 November 1992 flow unit. Both 1992 flow units are classified as vegetated surfaces using our classification criteria (Fig. 7b). This is consistent with the moderate cross-polarization backscatter from these flow units at Land P-bands (Fig. 7a). If the flow characterized by bare surface scattering signatures is younger than the flows erupted in late 1992, it would show that our technique is well able to distinguish the relative age of volcanic flows.

To determine the likelihood that the bare surface identified on the lower slope of NE Valley (Fig. 7) is a post-1992 flow, we examined the volcanic activity records maintained by the Smithsonian Institution. Eruptions from Main crater are the main source for lava and pyroclastic flows down NE valley (inset, Fig. 1). In January and in October 1994, lava flows from South crater reaching the sea were reported for SE Valley (Fig. 3; Smithsonian Institution, 1994). However, between the major eruptions in late 1992 and late 1996, ac- tivity remained low at Main crater and no significant lava or pyroclastic flows were reported for NE valley. During October and November 1996, five strong phases of activity occurred at Main crater at intervals of 7-12 days; four of them were preceded or accompanied by moderate activity at South crater (Fig. 2a; Smithsonian Institution, 1996). The first four phases at Main crater progressively increased in strength from early October to 10 November, and were accompanied by a large buildup in seismic activity (Fig. 2b; Smithsonian Institution, 1996). Like the strongest phase of the 1992 eruption, the first four eruptive phases at Main crater produced pyroclastic flows and lava flows in NE Valley. Thus, the bare surface area on the lower slope of NE Valley identified from the 17 November 1996 polarimetry and L-Band scattering signatures on the lower slope of NE Valley (Fig. 7) quite possibly represents a lava flow erupted during one of the first four strong eruptive phases from Main crater. If this is correct, that flow was younger than two weeks old when it was imaged. Its scattering signature stands in contrast to the vegetated surface signatures determined for the late 1992 lava flows (Fig. 7b). Plausible alternative explanations are (1) that the area identified as bare surface is a portion of the younger 5-6 November 1992 flow unit that somehow remained unvegetated while most of the 1992 lava flows became vegetated over the ensuing four years, or (2) the two underlying flows are very rough bare surfaces which generate significant depolarization and cross-polarized backscatter in the Pand L-band polarimetry (Fig. 7a). Although we know that flows in SE valley remained unvegetated for a year or more following eruption (e.g. Fig. 3), we think the first of the alternative explanations is unlikely. We emphasize that the lobate shape of the northern edge of the bare surface area (Fig. 7) is consistent with the outline of a lava flow unit. Evaluation of the second of the alternative explanations requires further ground truth information. 


\section{Conclusions}

The simple surface classification scheme based on scattering mechanism information extracted from AIRSAR L-Band polarimetry proved effective in distinguishing bare surfaces from forest cover over Manam volcano. Using data obtained on 17 November 1996, we were able to identify an apparently recent lava flow on the lower slope of the NE Valley that might have been erupted during the early stages of the large, late-1996 eruption sequence. We were also able to show using the AIRSAR polarimetry that lava flows erupted in the NE valley in 1992 appear to have become revegetated during the four years prior to data acquisition. We suggest that fully polarimetric SAR will be sensitive to scattering mechanism changes caused by volcanic resurfacing processes such as lava and pyroclastic flows. These data should therefore also be useful in mapping debris flows, as deposits and volcanic landslides. In terms of rapid response to volcanic eruptions: 1) Airborne or satellite polarimetric SAR technologies will have distinct operational advantages over optical remote sensing methods where persistent clouds, ash or smoke obscure the terrain during and after large eruptions, and 2) surface cover maps showing the distribution of eruptive products can be obtained quickly from a single acquisition of SAR polarimetry and analyzed with the methods discussed in this work, which are based on the physics of radar backscatter.

Acknowledgements. This research was supported by National Aeronautics and Space Administration (NASA) grants NAG58848 and NAG5-13731 (JKW), and Earth System Science Fellowship award NGT5-30374 (KRC). We thank F. Guzzetti for comments on an earlier version of the manuscript, and R. Lanari for helpful suggestions which improved the submitted version. LDEO contribution no. 6603.

Edited by: M.-C. Llasat

Reviewed by: R. Lanari and another referee

\section{References}

Boerner, W.-M., Mott, H., Lüneberg, E., Livingstone, C., Brisco, B., Brown, R. J., and Paterson, J. S.: Polarimetry in radar remote sensing: Basic and applied concepts, Ch. 5 in: Manual of Remote Sensing, Vol. 2 (3rd. Ed.), edited by Henderson, F. M. and Lewis, A. J., Am. Soc. Photogr. and Rem. Sens., 271-358, 1998.

Cloude, S. R. and Pottier, E.: A review of target decomposition theorems in radar polarimetry, IEEE Trans. Geosci. Rem. Sens., 34, 498-518, 1996.

Cloude, S. R. and Pottier, E.: An entropy based classification scheme for land applications of polarimetric SAR, IEEE Trans. Geosci. Rem. Sens., 35, 68-78, 1997.

Czuchlewski, K. R., Weissel, J. K., and Kim, Y.: Polarimetric synthetic aperture radar study of the Tsaoling landslide generated by the 1999 Chi-Chi earthquake, Taiwan, J. Geophys. Res., 108(F1), 6006, doi:10.1029/2003JF000037, 2003.

Huynen, J. R.: Measurement of the target scattering matrix, Proc. IEEE, 53, 936-946, 1965.

Kim, Y. and van Zyl, J.: Comparison of forest estimation techniques using SAR data, Proc. IGARSS2001 Conf., IEEE (CDROM), 2001.

Le Toan, T., Beaudoin, A., Riom, J., and Guyon, D.: Relating forest biomass to SAR data, IEEE Trans. Geosci. Rem. Sens., 30, 403411, 1992.

Nghiem, S. V., Bourgeau, M., Kong, J. A., and Shin, R. T.: Polarimetric remote sensing of geophysical media with layer random medium model, Ch. 1, in: Progress in electromagnetic research, Vol. 3, Polarimetric remote sensing, edited by Kong, J. A., Elsevier, Amsterdam, 1-73, 1990.

Nghiem, S. V., Yueh, S. H., Kwok, R., and Li, F. K.: Symmetry properties in polarimetric remote sensing, Radio Science, 27, 693-711, 1992.

Palfreyman, W. D. and Cooke, R. J. S.: Eruptive history of Manam volcano, Papua New Guinea, in: Volcanism in Australasia, edited by Johnson, R. W., Elsevier, Amsterdam, 117-131, 1976.

Smithsonian Institution, Manam: Bulletin of the Global Volcanism Network, 17(11), http://www.volcano.si.edu/reports/ bulletin/index.cfm, 1992.

Smithsonian Institution, Manam: Bulletin of the Global Volcanism Network, 19(11), http://www.volcano.si.edu/reports/ bulletin/index.cfm, 1994.

Smithsonian Institution, Manam: Bulletin of the Global Volcanism Network, 21(12), http://www.volcano.si.edu/reports/ bulletin/index.cfm, 1996.

Valenzuela, G. R.: Theories for the interaction of electromagnetic and ocean waves - A review, Boundary Layer Meteorol., 13, 61$85,1978$.

Zebker, H. A., van Zyl, J. J., and Held, D. A.: Imaging radar polarimetry from wave synthesis, J. Geophys. Res., 92, 683-701, 1987. 